Documentation et bibliothèques

DOCUMENTATION BIBLIOTHÈQUES

\title{
Un trésor à découvrir : les publications gouvernementales fédérales destinées aux bibliothèques scolaires et publiques
}

\section{Louise Tousignant}

Volume 39, numéro 1, janvier-mars 1993

URI : https://id.erudit.org/iderudit/1028599ar

DOI : https://doi.org/10.7202/1028599ar

Aller au sommaire du numéro

Éditeur(s)

Association pour l'avancement des sciences et des techniques de la documentation (ASTED)

ISSN

0315-2340 (imprimé)

2291-8949 (numérique)

Découvrir la revue

Citer cet article

Tousignant, L. (1993). Un trésor à découvrir : les publications gouvernementales fédérales destinées aux bibliothèques scolaires et publiques. Documentation et bibliothèques, 39(1), 31-32.

https://doi.org/10.7202/1028599ar

Tous droits réservés (c) Association pour l'avancement des sciences et des techniques de la documentation (ASTED), 1993
Ce document est protégé par la loi sur le droit d'auteur. L'utilisation des services d'Érudit (y compris la reproduction) est assujettie à sa politique d'utilisation que vous pouvez consulter en ligne.

https://apropos.erudit.org/fr/usagers/politique-dutilisation/ 


\title{
Un trésor à découvrir : les publications gouvernementales fédérales destinées aux bibliothèques scolaires et publiques
}

\author{
Louise Tousignant \\ Service du programme de dépôt \\ Groupe Communication Canada - Édition \\ Approvisionnements et Services Canada
}

En période de restrictions budgétaires, toute bibliothèque chercheà maximiser son budget. II faut savoir composer avec moins d'argent mais offrir un service de même niveau sinon meilleur. Cette situation s'observe particulièrement dans les bibliothèques publiques et scolaires. Comment offrir des nouveautés à l'aide de matériel, beau, bon, pas cher? On écarte souvent du revers de la main, par méconnaissance ou par manque de publicité, cette ressource que sont les publications gouvernementales fédérales'. En effet, ces dernières reçoivent les quolibets acerbes de toutes sortes: non attrayantes, mornes, ennuyeuses ou trop techniques pour ne nommer que ceux-là. Cette vision se révèle en grande partie inexacte. II est vrai que le gouvernement fédéral publie des rapports laborieux, des documents administratifs ou des titres techniques, mais il n'en demeure pas moins vrai qu'il met à la disposition des Canadiens une panoplie de publications destinées aux enfants, aux adolescents et au public en général. Qui plus est, ces livres sont généralement gratuits ou vendus à prix raisonnables.

Comme dans le secteur privé, ces publications se veulent culturelles, informatives et utiles. Le personnel de la documentation oeuvrant dans le développement des collections dans les bibliothèques publiques et scolaires découvrira avec plaisir un éventail de publications fort intéressantes pour les lecteurs attirés par des sujets aussi variés que l'environnement ou l'astronomie.

\section{Jeunes et adolescents}

Les jeunes de 12 à 15 ans voyageront au tiers monde avec les publications de l'Agence canadienne de développement international (ACDI). Esquisse de pays est une série d'affiches sur des pays en voie de développement. Chaque affiche présente la géographie physique et humaine, les dimensions culturelles, sociopolitiques et économiques d'un pays. Graphiques, illustrations et tableaux complètentle texte. Àtitre d'exemples, la Thaïlande et le Pakistan.

Publiée trois fois par année scolaire, la revue Sous un même soleil traite d'un thème par exemple la terre, la santé, les villes. Elle est présentée sur du papier glacé en couleur avec de belles illustrations. Cette revue peut servir autant à la lecture personnelle qu'à la lecture dirigée.

Un dessin pour Tara (1989) est un conte illustré pour les 4 à 7 ans. II est disponible en petit format pour la lecture individuelle ou en grand format pour l'heure du conte.

Justice Canada offre Le secret du petit cheval (1989). Ce livre renseigne les jeunes du primaire sur les abus sexuels par le biais d'une histoire illustrée. L'enfant apprendra à respecter les confidences, mais il apprendra également qu'il n'a pas à respecter le secret de l'exploitation sexuelle. Un poster à l'effigie des quatre personnages du livre est également disponible.

Pêches et Océans Canada propose Conte du vendredi (1991). Ce livret sensibilisera les élèves du $2^{e}$ cycle primaire à l'importance de protéger les lacs, les fleuves, les rivières et les océans. Ce récit didactique contient des jeux-questionnaires, des jeux d'esprit et des illustrations à colorier qui permettent aux enfants d'élargir leur champ de connaissance quant à l'industrie de la pêche. Les lecteurs du $2^{\circ}$ cycle secondaire, pour leur part, apprécieront certainement consulter la collection La faune de l'arrière-pays pour leurs travaux en géographie ou en biologie. Publié par le Service canadien de la faune, chaque numéro présente un animal vivant au Canada dont on décrit les moeurs, l'habitat et la répartition géographique. Des références et des croquis complètent la description de la faune canadienne. Cette série est d'ailleurs publicisée à la télévision par des capsules éclair.

Votre bibliothèque se situe dans un milieu bilingue? Qu'à cela ne tienne, le Commissariat aux langues officielles saura sensibiliser vos jeunes à la réalité linguistique. Dans les aventures de Julie et de Daniel, deux personnages à l'allure d'oiseaux propulsés dans un monde fantastique, les enfants de 5 à 7 ans découvriront qu'on peut s'entendre même si on ne parle pas la même langue. Cette bande vidéo intitulée Le mur magique (1990) est accompagnée d'une affiche et d'un guide pour le personnel enseignant. Les professeurs d'anglais langue seconde apprécieront les posters en couleurs. Nos amis à plumes donne les noms français et anglais d'oiseaux d'Amérique du Nord et $L e$ français et l'anglais... dans près de la moitié des pays du monde présente, sur un fond de ballons, les drapeaux des pays dans lesquels nos deux langues officielles sont communément utilisées. Enfin, le personnel enseignant de $5^{\mathrm{e}}$ secondaire est probablement déjà familier avec l'Agenda (annuel) du Commissariat. Cet agenda est non seulement pratique pour l'étudiant, mais il abonde en renseignements sur le Canada. Dans une édition précédente, on

1. Toute publication produite aux frais du gouvernement fédéral. 
pouvait lire que «le mot Canada n'est ni d'origine française ni d'origine anglaise : on croit qu'il vient du mot iroquois kanata qui signifie village».

Les albumsà colorier Me voici! (1990)* de Santé et Bien-être social Canada et L'ours prudent (1989) de la Gendarmerie royale du Canada informeront les jeunes tout en développant leur talent de dessinateur. Me voici! traite des soins apportés à un nouveau-né tandis que L'ours prudent présente les mesures de sécurité à prendre dans diverses situations. Dans les deux cas, un texte accompagne chaque image à colorier.

Par des points à relier, des mots à compléter, des jeux d'associations et des jeux de découpage et de collage, les jeunes découvriront le monde des dinosaures en parcourant les 48 pages du livre Qu'est-ce qu'un dinosaure? (1991)* publié par le Musée canadien de la nature.

L'écolo en herbe (1991) propose également du découpage et du collage, mais il suggèreaussi des activités à réaliser à la maison comme mettre en pratique les $4 \mathbf{R}$ (réduction, réutilisation, recyclage, récupération). Par cette publication, Environnement Canada conscientise les jeunes à la fragilité de notre environnement et à sa sauvegarde par l'entremise de son Plan vert.

Vous connaissez sans doute le populaire Palmarès (1991). C'est un guide des programmes et des services du gouvernement fédéral pour la jeunesse. Les adolescents trouveront plus de 100 programmes et services dans le domaine de l'éducation, de la formation, de l'emploi, des affaires et des voyages.

\section{Personnel enseignant}

Statistique Canada proposait en 1991 sa Trousse de l'enseignant surle recensement de 1991. Cette dernière a été conçue dans le but de sensibiliser les élèves au recensement et d'en faire comprendre son importance. Cette trousse suggère six activités d'apprentissage à l'aide de modules à réaliser en classe et à la maison pour les jeunes des niveaux primaire et secondaire.
Dans la même veine, le Secrétariat d'État du Canada met à votre disposition sa publication Symboles canadiens $(1991)^{\star}$. Les élèves du secondaire en apprendront davantage sur l'emblème floral, les armoiries, le drapeau, l'origine du nom de chaque province et de chaque territoire.

\section{Public en général}

Les futurs parents découvriront avec plaisir Votre enfant et vous (1992)*. Ce best-seller depuis plus de cinquante ans porte sur les préoccupations premières des parents allant des soins nécessaires pendant la grossesse à l'alimentation du bébé en passant par la sécurité et les premiers soins.

Deux brochures également publiées par Santé et Bien-être social Canada et destinées aux parents compléteront la lecture de ce guide. II s'agit de Mon enfant au soleil : guide des parents (1992) et de L'environnement et la santé de votre enfant : comment protéger votre enfant des substances dangereuses à la maison (1992).

La question de l'environnement préoccupe tous et chacun, vous comme moi. Le bureau écologique : conseils pratiques pour rendre votre bureau respectueux de l'environnement (1992) fournit des idées et des adresses afin de diminuer et d'éliminer les déchets le plus harmonieusement possible. Des exemples concrets de compagnies aussi connues que Bell Canada avec son opération «Zéro déchet» rehaussent ce document de 48 pages.

Ce que nous pouvons faire pour notre environnement : mille et une choses à faire (1990) donne des trucs, des habitudes de vie à changer à la maison, à l'école, au travail et même en magasinant. Tout comme pour Le bureau écologique, on y fournit des adresses pour obtenir de plus amples renseignements sur la gestion des déchets.

Un autre domaine pour lequel vous ferez probablement une découverte est le secteur résidentiel. La Société canadienne d'hypothèques et de logement publie en effet une foule de documents dont Proté- gez votre résidence contre le vol (1988)", Amélioration des espaces extérieurs des vieilles maisons $(1983)^{\star}$, et Logements pour Canadiens ágés : nouveaux mécanismes financiers et mode d'occupation (1987).

Du côté de l'énergie, vous serez sans doute ravis de prendre connaissance des publications et objets offerts par Énergie, Mines et Ressources Canada. II y a, entre autres: Le guide des économies au volant (1991), Le calculateur des économies au volant (1992), Dans un monde où le coût de l'énergie augmente sans cesse (signet), L'achat d'une maison R-2000(1991).

Comme dans le secteur privé, certaines publications fédérales remportent des prix. C'est le cas notamment de la publication Les secrets du Saint-Laurent: guide de météo marine. Cette dernière s'est vue décerner en 1992 le prix J.-Oscar Villeneuve par la Société de météorologie de Québec pour sa contribution exceptionnelle aux plans de la vulgarisation et de l'apport scientifique. Publiée par Environnement Canada, Les secrets du SaintLaurent est destinée aux plaisanciers désireux de se familiariser avec ce plan d'eau. Cette publication existe en version imprimée (1992)* et sur bande vidéo (1992)*.

Et si vos usagers troquaient le bateau pour l'avion, il pourrait s'avérer utile pour eux de lire Prenez l'air averti(1991). Dans ce document, l'Office national des transports expose les façons d'éviter les problèmes de déplacement par avion.

La documentation présentée ici ne reflète qu'un échantillon de toutes les publications gouvernementales fédérales disponibles sur le marché. En y prenant le temps, nous nous apercevons que nous sommes entourés de publications gouvernementales. Nous l'oublions parfois, mais le Répertoire des codes postaux au Canada en est même une! C'est à espérer que les bibliothèques scolaires et publiques apprendront davantage à tirer profit de cette littérature souvent méconnue des bibliothécaires et du public en général.

\footnotetext{
* Les titres des publications vendues sont suivis d'un astérisque. Dans tous les autres cas, il s'agit de publications distribuées gratuitement.
} 